\title{
Automatic detection of polyomavirus particles with local texture indicators
}

\author{
M.C. Proença***, J.F. Moura Nunes *** and A.P. Alves de Matos******* \\ *Laboratório de Óptica, Lasers e Sistemas, Faculdade de Ciências da Universidade de Lisboa, Ed. C8, 1749-016 \\ Lisboa \\ **Centro de Estudos do Ambiente e do Mar - Polo de Lisboa, Faculdade de Ciências da Universidade de Lisboa, \\ Ed. C8, 1749-016 Lisboa \\ ***Laboratório de Microscopia Electrónica, Instituto Português de Oncologia, Rua Prof. Lima Basto, 1099-023 \\ Lisboa \\ ****Anatomia Patológica, Centro Hospitalar de Lisboa Central - HCC, Rua da Beneficência 8, 1069-166 \\ Lisboa, Portugal and Centro de Investigação Interdisciplinar Egas Moniz (CiiEM), Quinta da Granja, Monte de \\ Caparica, 2829-511 Caparica, Portugal
}

A fully automatic approach to locate polyomavirus particles in images of transmission electronic microscopy is presented, that can localize intact particles, many damaged capsids and an acceptable percentage of superposed ones; the performance is quantified in 25 electron micrographs containing nearly 390 particles, compared to the interpretation of two independent electron microscopy experts. As these particles often does not have a welldefined edge, in highly textured backgrounds (Figure 1) the initial guess of candidate points from local entropy proportion measures retains a large set of points, in order to avoid false negatives.

Based in human criteria - focused on the pattern generated by the capsomeres typical of polyomavirus surface, this approach uses indicators calculated from the local co-occurrence matrix of grey levels [1] to assess the textured pattern and prune the initial set of candidates; in some more complicated backgrounds about $2-10 \%$ of the elements will survive. A restricted set of the points accepted (Figure 2) is used to evaluate typical average and variance and reduce the set of survivors accordingly. These intermediate points are locally evaluated using i) a statistical index concerning the radiometric distribution of a circular neighborhood around the centroid of each candidate, and ii) a structural index resuming the expected morphological characteristics of eight radial intensity profiles encompassing the area of the possible particle. All the parameterization is based on the particle expected dimensions.

This hierarchical approach attains 95\% efficiency in the detection of intact virus particles, tolerating a certain lack of differentiation in the borders and a certain amount of shape alterations. Only 5 to 7\% (according to the reference used) of the intact particles are missed by the algorithm - cases of particles with abnormal radial structures, particles with different degrees of agglutination or particles showing empty (or with different degrees of permeation) capsids. Superposed particles are detected with a success rate of $71 \%$ to $77 \%$. In the case of distorted and/or doubtful particles, a correct detection of $72 \%$ is attained; these particles are the main source of differences between experts (a difference of $48 \%$ between both interpretations).

This kind of algorithm is "tailored" for one virus family particle, in this case, the polyomavirus; it can be parameterized for different working magnifications, but cannot be applied to different virus, that has different texture, structure and typical radiometry characteristics. We believe that similar results can be obtained in other virus families with the necessary adaptations.

\section{References}

1. Haralick R.M. et al., IEEE Transactions on Systems, Man and Cybernetics, 3:610-621, 1973. 

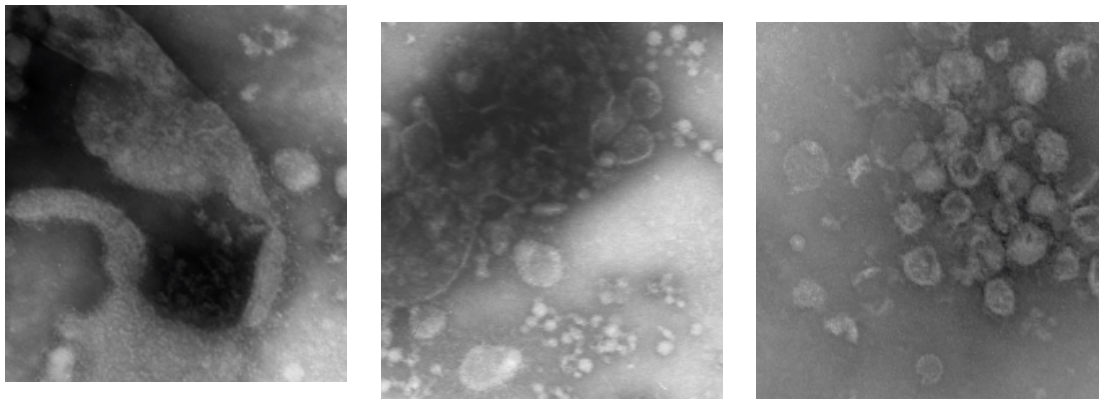

Figure 1. Details of the variety of biologic debris and fluid traces in the background.

a)

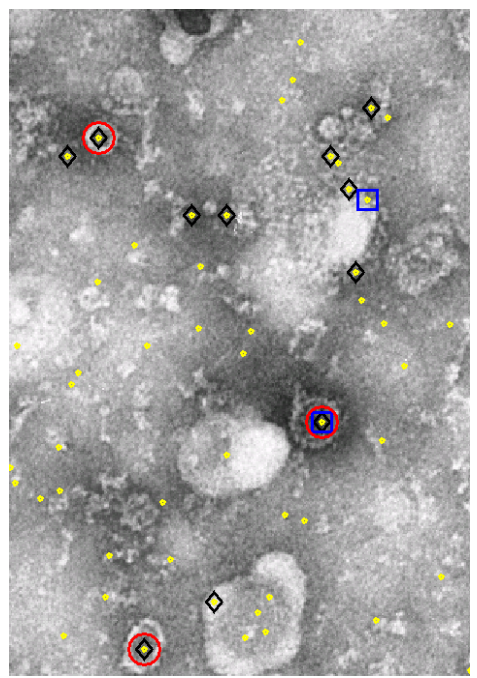

b)

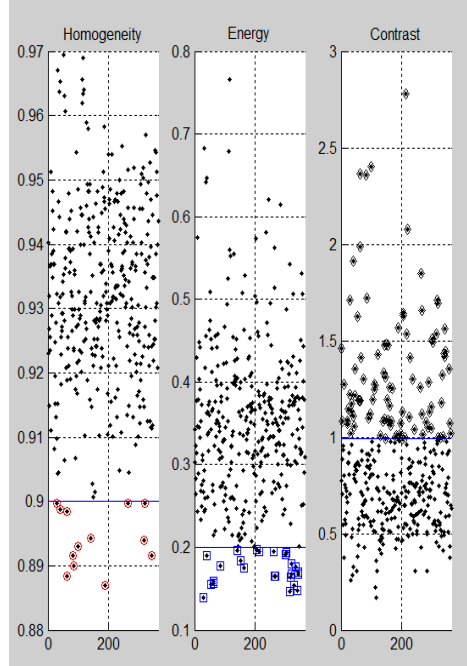

Figure 2. It's the intersection of the three criteria - homogeneity, energy and contrast - that localizes the majority of interesting points; we can see in the sub-image (a) that the unique point of this area satisfying the low homogeneity criteria (circle red), low energy (square blue) and high contrast (diamond black) is a virus particle. Points in yellow don't satisfy any of the criteria. The thresholds in b) are related to the variance and entropy of the initial image.

a)

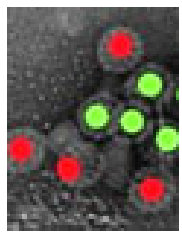

b)

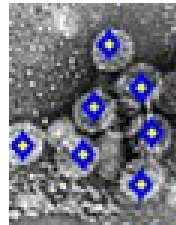

c)

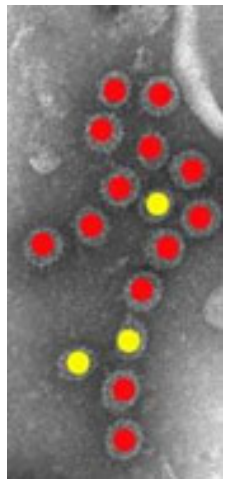

d)

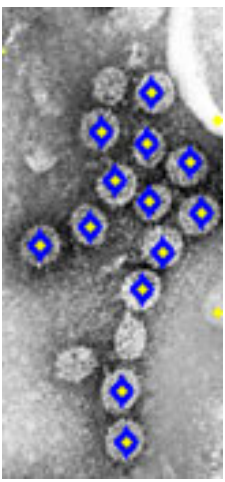

Figure 3. (a) and (c) Examples of reference data marked by trained observers; intact particles (red dots), semitransparent (green) and distorted/doubtful virus particles (yellow). (b) and (d) Corresponding results of the automatic procedure, with the detections marked in blue. 